\title{
Selective amplification of frequency comb modes via optical injection locking of a semiconductor laser: influence of adjacent unlocked comb modes
}

\author{
David S. Wu*, David J. Richardson, and Radan Slavík \\ Optoelectronics Research Centre, University of Southampton, Southampton, SO17 1BJ, \\ United Kingdom;
}

\begin{abstract}
Optical injection locking can be used to isolate and amplify individual comb modes from an optical frequency comb (OFC). However, it has been observed that for narrow spaced OFCs (e.g. $250 \mathrm{MHz}$ ), the adjacent comb modes are still present in the output of the locked laser. These residual modes experience some amplification relative to the injected signal, however the gain is significantly less than for the locked mode. We report the measurement of this sidemode amplification for a semiconductor laser injection locked to a $250 \mathrm{MHz}$ spaced OFC. It was found that this amplification can be well suppressed by tuning the frequency difference between the free running laser and the OFC mode it was locked to. The sidemode amplification was then investigated numerically by solving the laser rate equations under optical injection. It was found that the main contribution to the sidemode amplification was due to phase modulation induced by the residual comb modes. The detuning dependent suppression occurs due to destructive interference between pairs of equidistant comb modes.
\end{abstract}

Keywords: Optical frequency combs; optical injection locking

\section{INTRODUCTION}

Optical frequency combs (OFCs) provide a direct link between the optical and radio frequency domains which has given rise to many applications in time and frequency metrology and spectroscopy. ${ }^{1}$ However, their ability to generate many distinct comb modes with a fixed frequency spacing between them also gives rise to applications in other fields such as telecommunications. For example, spectral efficiency in fibre optic communications can be improved by using coherent optical orthogonal frequency division multiplexing (CO-OFDM) which takes advantage of the coherence between comb modes to transmit data using multiple sub-channels with very small or no guard bands. ${ }^{2-4}$ This is achieved by demultiplexing an OFC into its individual comb modes, separately modulating data onto each mode and multiplexing them together. In a similar operating principle, pulse shaping can also be achieved by varying the amplitude and phase of each individual comb mode to generate the desired waveform (this is known as line-by-line pulse shaping). ${ }^{5-7}$

Optical injection locking is a technique where a 'slave' laser is forced to adopt the frequency of a 'master' laser by transmitting some light from the master laser into the slave laser cavity. ${ }^{8}$ This technique can also be used to process OFCs by isolating and amplifying individual comb modes. Since the slave laser retains its white noise properties, injection locking can amplify individual comb modes with very high optical signal to noise ratios (OSNR). This is in contrast to traditional optical amplifiers, e.g. erbium doped fibre amplifiers and semiconductor optical amplifiers, which always add additional broadband noise. Injection locking can also allow for the use of narrowly spaced $\mathrm{OFCs},{ }^{9,10}$ e.g. those generated by repetition rate and carrier envelope offset stabilised mode-locked lasers. The benefit of using these OFCs is that they can generate a very wide bandwidth of comb modes (over an octave) which is desirable for high repetition rate radio frequency generation ${ }^{11}$ and $\mathrm{THz}$ generation. ${ }^{12}$

We have previously demonstrated long-term injection locking of semiconductor lasers to a $250 \mathrm{MHz} \mathrm{OFC}$ by using small locking ranges and incorporating a slow feedback system to compensate for frequency drifting. ${ }^{13}$ Despite injection locking to only a single comb mode, some amplification of adjacent comb modes was observed

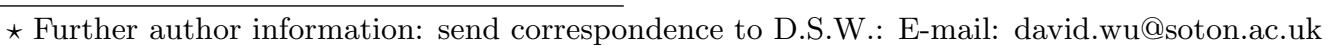


relative to their injected intensities. However, the gain experienced by the sidemodes was significantly less than that experienced by the locked mode. This effect was also previously observed in Ref. 14 . In this report, we experimentally measure the sidemode amplification in our system (Section 2). In Section 3, we further investigate the effect by numerically solving the laser rate equations with an optically injected OFC.

\section{OPTICAL INJECTION LOCKING - SIDEMODE AMPLIFCATION}

Optical injection locking occurs if the (angular) frequency detuning $\left(\Delta \omega=\omega_{0}-\omega_{F R}\right)$ between the free running slave laser $\left(\omega_{F R}\right)$ and the master laser $\left(\omega_{0}\right)$ is smaller than the locking range $\left(\Delta \omega_{L R}\right)$. The size of this locking range is proportional to the square root of the injection ratio, $\sqrt{I_{S} / I_{0}}$, where $I_{S}$ and $I_{0}$ are the intensities of the slave and master respectively. Optical injection locking of a cw slave laser to a cw master laser is a well established technique; for further details the reader is directed to Ref. 8 and specifically for semiconductor lasers, Ref. 15 and 16.

In the case of using an $\mathrm{OFC}$ as the master laser, the slave laser can behave as a selective amplifier by injection locking to only a single comb mode. This is achieved by using low injection ratios (where $I_{0}$ is now the intensity per comb mode) such that the locking range is smaller than the comb mode spacing. However, despite being locked to only a single comb mode, it was observed that the nearby injected comb modes may experience some gain as they travel inside the laser medium. In this section we report the measurement of the sidemode amplification.

\subsection{Experimental set-up}

The sidemode amplification of a discrete mode ${ }^{17}$ semiconductor laser injection locked to a $250 \mathrm{MHz}$ spaced OFC was measured. The OFC was a mode-locked fibre laser which was stabilised in both repetition rate and carrier envelope offset frequency, with a centre wavelength of approximately $1560 \mathrm{~nm}$. The experimental set-up is shown in Figure 1. A $50 \mathrm{GHz}$ passband arrayed waveguide grating (AWG) was used to loosely filter the comb such that only a small fraction of the OFC was injected into the slave laser. The injected power was controlled using a variable attenuator and the polarisation was aligned with the semiconductor slave laser using a polarisation controller. The semiconductor slave laser had no isolator and optical injection was achieved using a fiberised 3-port optical circulator. A slow feedback loop $(<30 \mathrm{~Hz})$ acted on the slave laser driving current to keep it locked long term ${ }^{13}$ by keeping $\Delta \omega$ at a fixed value to compensate for slow drifts in the slave laser free running frequency.

A reference laser (also a discrete mode semiconductor laser) was locked to the OFC using a purely electronic optical phase lock loop at an offset frequency of $30 \mathrm{MHz}$. The reference laser was combined with the injection locked slave laser to generate a $30 \mathrm{MHz}$ beat signal on a $800 \mathrm{MHz}$ bandwidth photoreciever. An RF spectrum analyser was used to measure the relative intensities of: the beat signal between the reference laser and the main mode of the slave laser (located at $30 \mathrm{MHz}$ ), and the beat signal between the reference laser and one of the adjacent the sidemode (located $280 \mathrm{MHz}$ ). It can be shown that this corresponds directly to the relative intensity of sidemode of the slave laser.

\subsection{Experimental results}

The sidemode amplification was measured for detunings across the locking range for injection ratios between -70 and $-55 \mathrm{~dB}$. The detuning was varied by tuning the locking position of the slave laser feedback loop by introducing an offset to the feedback error signal. The actual detuning was not able to be measured since the slave laser adopts the same frequency as the locked comb mode across the locking range. Hence the normalised detuning was estimated by assuming that the entire locking range was accessible by changing the locking position of the feedback loop. Linearity between detuning and the locking position was also assumed because we are using a semiconductor slave laser with a linewidth enhancement factor $(\alpha)$ of approximately 5 . This is large enough to make the feedback error signal approximately linear with detuning. ${ }^{13}$

Figure 2 shows the relative sidemode intensity across the locking range for different injection ratios. The sidemode amplification can be calculated by the difference between the injection ratio and the relative sidemode intensity at the output of the slave laser. Figure 2 shows that while the sidemode is at least $30 \mathrm{~dB}$ smaller than the 


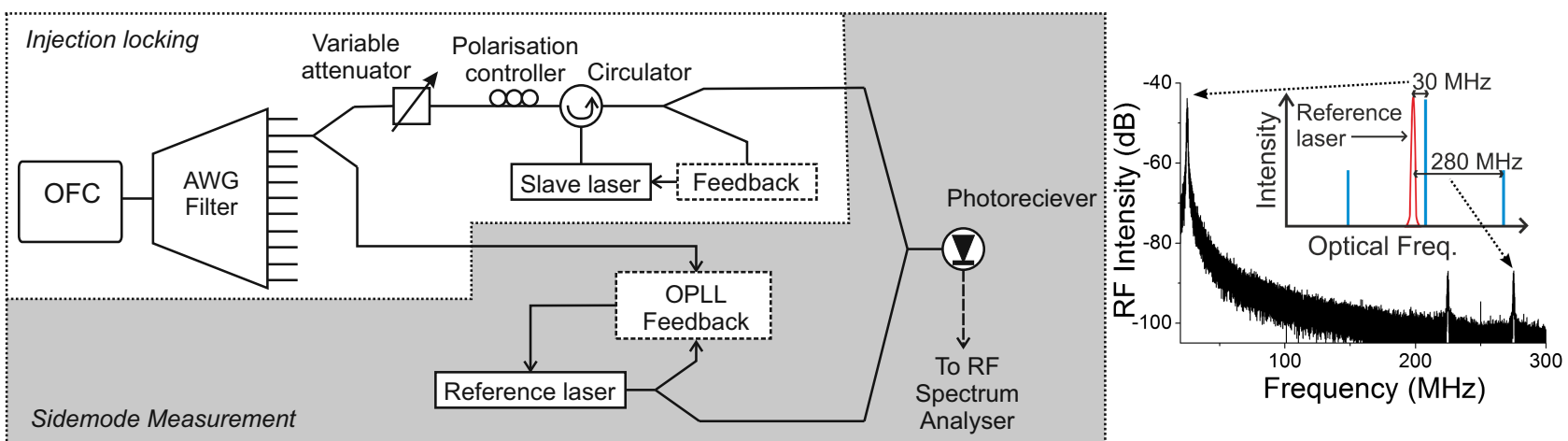

Figure 1: The experimental set-up for measuring the sidemode amplification. OPLL: optical phase lock loop. The RF spectrum on the right shows an example measurement (injection ratio $=-65 \mathrm{~dB} ; \Delta \omega / \Delta \omega_{L R} \approx 0.25$ ). The inset is an illustration of the corresponding optical spectrum.

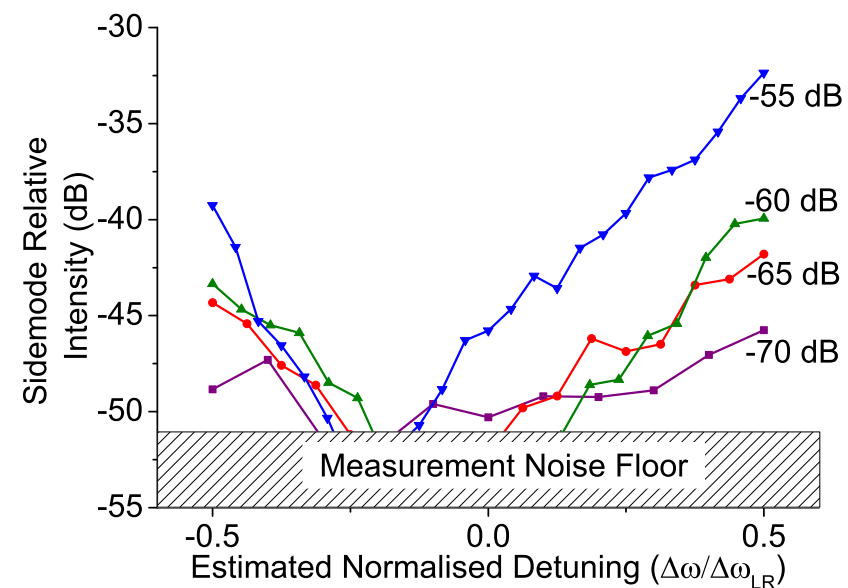

Figure 2: The measurement of sidemode amplification for an injection locked laser to a single mode of a 250 $\mathrm{MHz}$ spaced OFC for various injection ratios. The noise floor was $-52 \mathrm{~dB}$.

locked mode, it has still been amplified relative to its injected power. It should be noted that the measurement had a noise floor of $-52 \mathrm{~dB}$ which prevented us from measuring the maximum sidemode suppression.

A minimum sidemode intensity can be obtained by injection locking at a particular detuning (approximately -0.2 to 0.0 in Figure 2). A sidemode suppression of at least $-52 \mathrm{~dB}$ was attainable for all the injection ratios we used. The detuning dependence is not symmetric, with significantly less suppression on one end of the locking range. We believe that this feature is an artifact due to the assumptions we made to estimate the normalised detuning.

Figure 2 shows that the sidemode intensity is also dependent on the injection ratio. The larger the injection ratio, the larger the sidemode intensity is. This is not surprising, since a larger injection ratio increases the proximity of the adjacent comb modes to the locking range. However, regardless of the injection ratio used, the intensity of the sidemode can always be suppressed by tuning the value of $\Delta \omega$.

\section{THEORETICAL ANALYSIS}

\subsection{Rate equations}

In this section, we introduce a basic model based on the laser rate equations to further explore the sidemode amplification. The rate equations used here are closely based on those presented in Ref. 16, but we have modified the master laser to include multiple frequency modes (to simulate an OFC). We begin with the equation for the 
complex electric field of the slave laser under optical injection: ${ }^{16}$

$$
\frac{d}{d t} \tilde{E}_{S}(t)-\left(i \omega(N)+\frac{1}{2}\left[G(N)-\frac{1}{\tau_{p}}\right]\right) \tilde{E}_{S}(t)=\frac{K}{\tau_{i n}} \tilde{E}_{M}(t)
$$

where $\tilde{E}_{S}$ and $\tilde{E}_{M}$ are the complex electric fields of the slave and master laser; $\omega(N)$ and $G(N)$ are the angular optical frequency and modal gain per second of the slave laser which are dependent on $N$, the carrier density; $i$ is the imaginary number $\sqrt{-1} ; \tau_{p}$ is the photon lifetime in the laser cavity; $K$ is the coupling efficiency of the master signal into the slave laser; and $\tau_{i n}$ is the round trip time inside the laser cavity. The complex electric fields are defined as:

$$
\begin{aligned}
\tilde{E}_{S}(t) & =E_{S}(t) e^{i\left[\omega_{F R} t+\phi_{S}(t)\right]} \\
\frac{K}{\tau_{i n}} \tilde{E}_{M}(t) & =\sum_{j}\left\{E_{i n j}(t) e^{i\left[\omega_{j} t+\varphi\right]}\right\}
\end{aligned}
$$

where $E_{S}(t), \omega_{F R}$, and $\phi_{S}(t)$ are real valued amplitude, free running angular frequency and phase of the slave laser. The injected OFC is described in Equation (3), where the subscript $j$ is an index for each injected comb mode. For simplicity, we have set each comb mode at the input of the slave laser to have equal amplitudes given by $E_{i n j}$. For mode locked laser type OFCs, the comb modes have the same initial phase, $\varphi$, which we set to 0 . The angular frequency of each comb mode is given by $\omega_{j}$. Following similar steps to those taken in Ref. 16, the amplitude, phase and carrier density rate equations can be written as:

$$
\begin{array}{r}
\frac{d}{d t} E_{S}(t)=\frac{1}{2} G_{N}\left[N(t)-N_{t h}\right] E_{S}(t)+E_{i n j} \sum_{j} \cos \left(\delta \omega_{j} t-\phi_{S}(t)\right) \\
\frac{d}{d t} \phi_{S}(t)=\frac{1}{2} \alpha G_{N}\left[N(t)-N_{t h}\right]+\frac{E_{i n j}}{E_{S}(t)} \sum_{j} \sin \left(\delta \omega_{j} t-\phi_{S}(t)\right) \\
\frac{d}{d t} N(t)=R_{p}-\frac{N(t)}{\tau_{s}}-G_{N}\left[N(t)-N_{t h}\right] E_{S}(t)^{2}-\frac{1}{\tau_{p}} E_{S}(t)^{2}
\end{array}
$$

The definitions and values of the constants in Equations (4)-(6) are provided in Table 1. $\delta \omega_{j}$ is the detuning between each comb mode and the slave laser $\left(\omega_{j}-\omega_{F R}\right)$. The gain is assumed to be independent of wavelength since we only consider a small number of closely spaced comb modes. The injection ratio is defined as $E_{i n j} / E_{S 0}$, where $E_{S 0}$ is the steady-state electric field amplitude of the free running (no optical injection) slave laser.

Equations (4) and (5) show that the effect of injecting multiple comb modes into the laser is to introduce both amplitude and phase modulation into the slave laser. Laser feedback can then amplify these modulation effects which can result in sidemodes which are larger than their injected intensities i.e. sidemode amplification.

Table 1: Semiconductor laser parameters used in the rate equation modelling

\begin{tabular}{|l|l|}
\hline$G_{N}=8.1 \times 10^{-13} \mathrm{~m}^{3} \mathrm{~s}^{-1}$ & Differential gain \\
\hline$N_{t h}=1.7172 \times 10^{24} \mathrm{~m}^{-3}$ & Carrier density at threshold (includes cavity losses) \\
\hline$\alpha=5$ & Linewidth enhancement factor (amplitude-phase coupling) \\
\hline$K=0.2$ & Injected light coupling coefficient \\
\hline$\tau_{\text {in }}=6 \times 10^{-12} \mathrm{~s}$ & Laser cavity round trip time \\
\hline$R_{p}=1.7833 \times 10^{34} \mathrm{~s}^{-1}$ & Pump rate \\
\hline$\tau_{s}=2 \times 10^{-9} \mathrm{~s}$ & Carrier lifetime \\
\hline$\tau_{p}=2 \times 10^{-12} \mathrm{~s}$ & Photon lifetime \\
\hline
\end{tabular}




\subsection{Modelling results}

We first considered a total of 9 comb modes to be injected, with a comb spacing of $250 \mathrm{MHz}$. The free running frequency of the slave was chosen such that it would injection lock to the centre comb mode. We were unable to solve the rate equations analytically and hence they were solved numerically in the time domain and Fourier transformed into the frequency domain using FFT to calculate the relative intensities of the different output modes. This was done using a variable step, non-stiff, Runge-Kutta method.
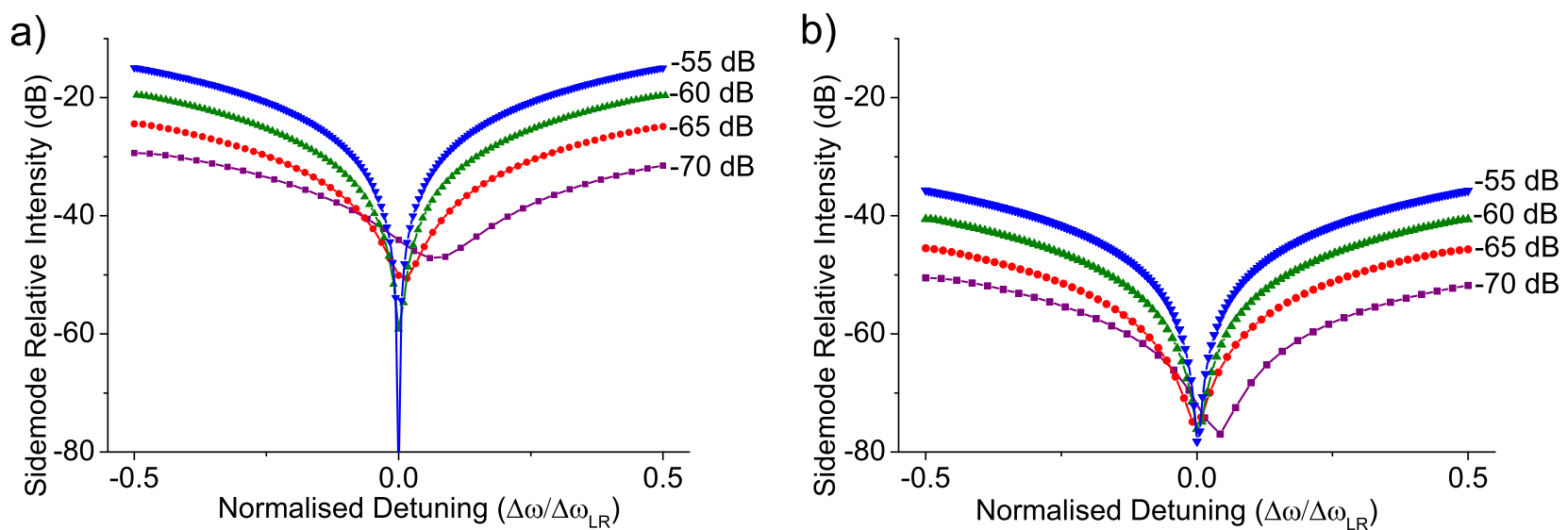

Figure 3: The calculated sidemode intensity relative to the main locked mode using various injection ratios for an injected signal consisting of 9 comb modes with a spacing of (a) $250 \mathrm{MHz}$, and (b) $2.5 \mathrm{GHz}$.

Figure 3a shows the calculated intensity of the higher-frequency adjacent mode relative to the locked slave mode. This was done by varying $\Delta \omega$ across the locking range (in steps of $0.5 \mathrm{MHz}$ ) and for various injection ratios $(-70$ to $-55 \mathrm{~dB})$. Similar to the experimental results, this shows that regardless of the injection ratio, $\Delta \omega$ can be tuned such that the sidemode intensity is at a minimum. For certain injection ratios, the sidemode intensity can even be smaller than its injected intensity. It can also be seen that at the edge of the locking range, the sidemode intensity is at a maximum. This maximum intensity increases for larger injection ratios. These trends are consistent with our experimental results.

There are also some discrepancies between the experimentally measured and numerically calculated results. The calculated results show only a slight asymmetry with the detuning, which is only noticeable at low injection ratios. Also, compared to the experimental results, the sidemode amplification is larger for the calculated results, with the relative intensity of the sidemode reaching as high as $-15 \mathrm{~dB}$. As previously mentioned, these discrepancies may be due to how we estimated the detuning, $\Delta \omega$, in the experiment, for example, we may not have been reaching the entire locking range in our experiment.

It is expected that by using an OFC with wider comb spacing, the sidemode amplification will be smaller for a given injection ratio. This is because the residual comb modes will be located further away from the locking range of the slave laser. This hypothesis is supported by Figure 3b, which shows the sidemode intensity using an increased comb spacing of $2.5 \mathrm{GHz}$. The maximum sidemode intensity, at the edge of the locking range, is approximately $20 \mathrm{~dB}$ less than for a $250 \mathrm{MHz}$ spaced OFC.

Further insight can be gained by looking at the solutions for $E(t)$ and $\phi(t)$ individually. For simplicity, the number of comb modes was reduced to three, with the slave laser being locked to the central mode. $E(t)$ and $\phi(t)$ are shown in Figures 4 and 5 for: detunings of 0 and $10 \mathrm{MHz}$, and comb spacings of $250 \mathrm{MHz}$ and $2.5 \mathrm{GHz}$. It was found that the slave laser will reach a quasi-steady state at a finite time after optical injection (approximately $50 \mathrm{~ns})$. In this state, the amplitude and phase of the locked slave laser can be described by:

$$
\begin{array}{r}
E_{S}(t)=E_{\text {locked }}+a(\Delta \omega, \Omega) \sin (\Omega t) \\
\phi_{S}(t)=\phi_{\text {locked }}+\Delta \omega t+b(\Delta \omega, \Omega) \cos (\Omega t)
\end{array}
$$



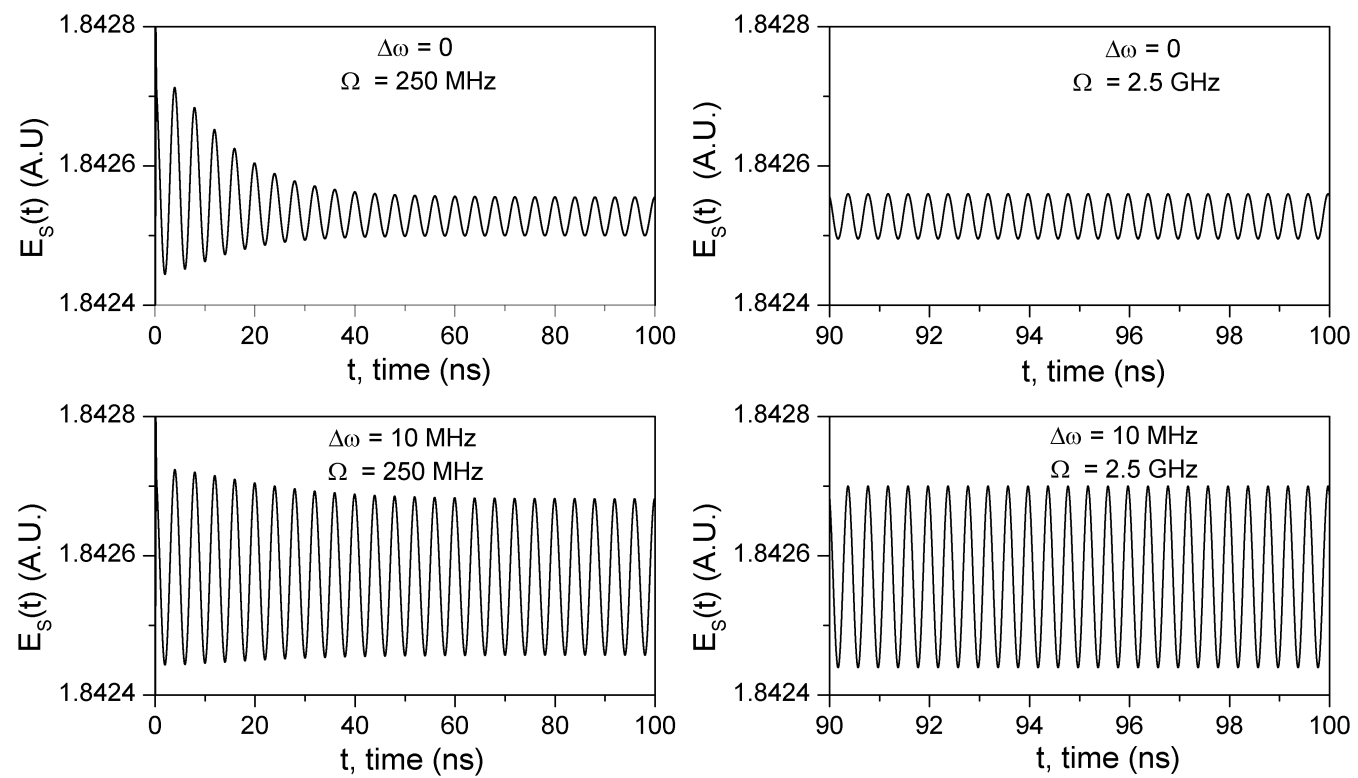

Figure 4: The slave laser electric field amplitude with 3 injected comb modes. Note that for $\Omega=2.5 \mathrm{GHz}, E_{S}(t)$ is only shown after the quasi-steady state was reached.
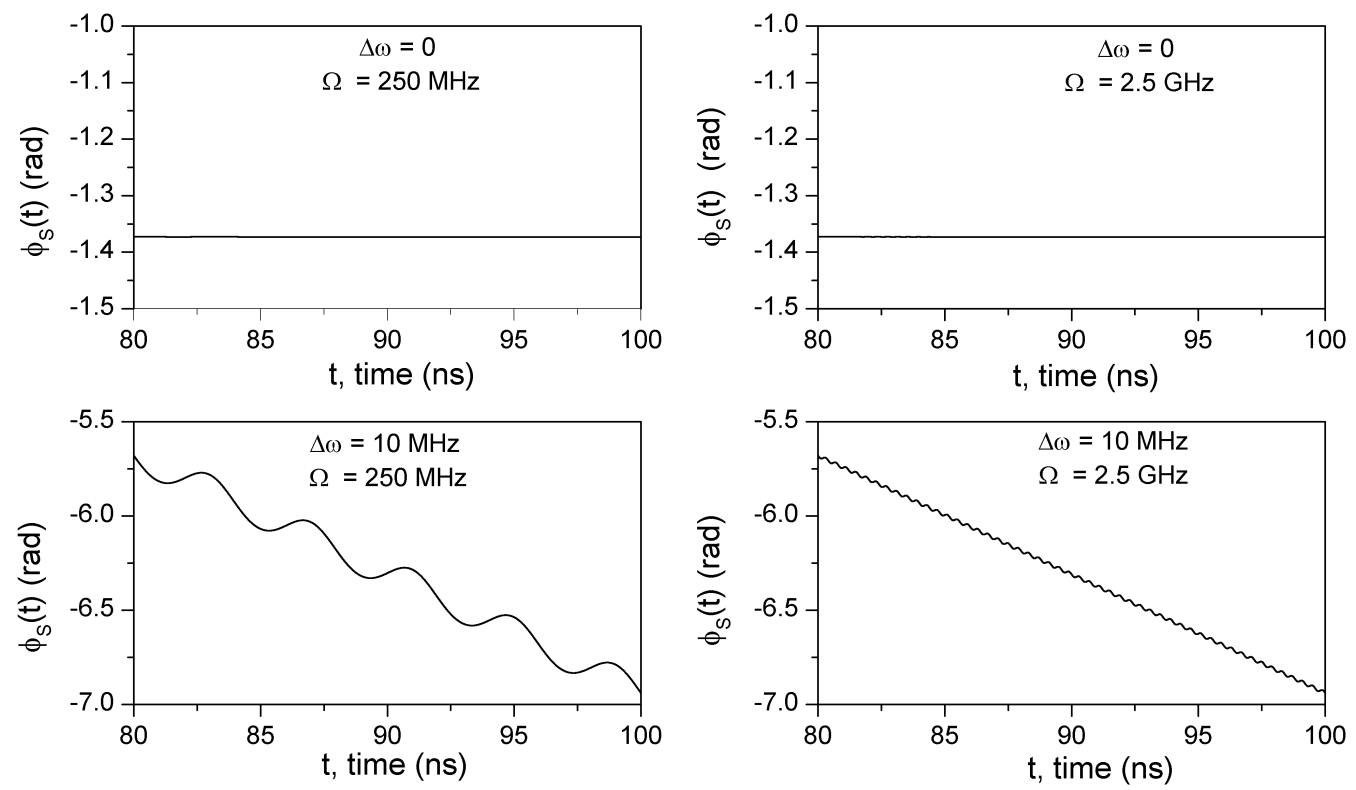

Figure 5: The slave laser phase with 3 injected comb modes after a quasi-steady state was reached.

The electric field amplitude, Equation (7), has a constant component, $E_{\text {locked }}$, and an amplitude modulation component given by the second term. The phase of the slave laser, Equation (8), also has a constant phase shift, $\phi_{\text {locked }}$, and a modulation component. In addition, it contains a linear phase component of $\Delta \omega t$ which shifts the frequency of the lasing mode to match the frequency of the comb mode it is locked to. The amplitude and phase modulation components of $E_{S}(t)$ and $\phi_{S}(t)$ have modulation depths of $a(\Delta \omega, \Omega)$ and $b(\Delta \omega, \Omega)$ which are dependent on $\Delta \omega$ and the comb spacing, $\Omega$. The modulations are sinusoidal with a frequency equal to $\Omega$.

The amplitude modulation depth, $a(\Delta \omega, \Omega)$ was found to increase both with $|\Delta \omega|$ and $\Omega$. This shows that for the parameters used, the injected comb will always introduce amplitude modulation regardless of the comb spacing or the detuning. 
The phase modulation depth, $b(\Delta \omega, \Omega)$, also increases with increasing $|\Delta \omega|$. However, the phase modulation can be heavily suppressed (by at least 3 orders of magnitude) by tuning $\Delta \omega=0$. This can be explained by Equation (5), where the terms involving these two residual comb modes can be written as:

$$
\sin ([\Delta \omega+\Omega] t+\phi(t))+\sin ([\Delta \omega-\Omega] t+\phi(t))
$$

These two sine terms destructively interfere as $\Delta \omega \rightarrow 0$, which suppresses their influence on phase. This was confirmed by injecting only two comb modes in the simulations. In this non-symmetric case, it was found that reducing the detuning had a negligible effect on $b(\Delta \omega, \Omega)$. Since Equation (4) involves cosine terms, this suppression does not take place with the electric field amplitude.

The phase modulation depth was also found to be inversely proportional to the comb spacing, in contrast to the amplitude modulation depth. The phase modulation therefore has behaviour which is consistent with Figure 3. We therefore believe that the phase modulation is the dominant contribution to the sidemode amplification.

The effects of increasing the injection ratio will be the subject of future investigation. It should be noted that for these simulations, it was assumed that all the comb modes had the same initial phase, which is the case for mode-locked laser type OFCs. However, this is not true for OFCs generated using phase modulation and therefore the same results cannot be assumed for these type of OFCs.

\section{CONCLUSION}

The amplification of residual comb modes after injection locking a semiconductor laser to an individual mode of an OFC has been investigated. We experimentally measured this amplification using a heterodyne technique and found that the maximum sidemode intensity increased with increasing injection ratios. However, the intensity of the sidemodes can be reduced to less than $-50 \mathrm{~dB}$ relative to the locked mode by tuning the frequency difference between the slave laser and the locked comb mode. This was true over the range of injection ratios over which we were able to achieve locking experimentally ( -70 to $-55 \mathrm{~dB})$.

These trends were confirmed by numerically solving the laser rate equations for a slave laser externally injected with an OFC. The equations show that the external injection of multiple comb modes introduces both amplitude and phase modulation of the slave laser, however the phase modulation was found to be the dominant contribution. The strong suppression of the sidemode amplification via detuning was found to correspond with destructive interference of the modulation contribution from pairs of injected comb modes when they are equidistant from the freqeuncy of the free running slave laser.

\section{ACKNOWLEDGMENTS}

This work was supported by the UK EPSRC through grants EP/K003038/1 and EP/H02607X/1 (Photonic Hyperhighway).

\section{REFERENCES}

[1] J. Ye, H. Schnatz, and L. W. Hollberg, "Optical frequency combs: From frequency metrology to optical phase control," IEEE Journal of Selected Topics in Quantum Electronics 9(4), pp. 1041-1058, 2003.

[2] A. D. Ellis and F. C. G. Gunning, "Spectral density enhancement using coherent WDM," Photonics Technology Letters, IEEE 17(2), pp. 504-506, 2005.

[3] Y. Xingwen, N. K. Fontaine, R. P. Scott, and S. Yoo, "Tb/s Coherent Optical OFDM Systems Enabled by Optical Frequency Combs," Lightwave Technology, Journal of 28(14), pp. 2054-2061, 2010.

[4] D. Hillerkuss, R. Schmogrow, T. Schellinger, M. Jordan, M. Winter, G. Huber, T. Vallaitis, R. Bonk, P. Kleinow, F. Frey, M. Roeger, S. Koenig, A. Ludwig, A. Marculescu, J. Li, M. Hoh, M. Dreschmann, J. Meyer, S. Ben Ezra, N. Narkiss, B. Nebendahl, F. Parmigiani, P. Petropoulos, B. Resan, A. Oehler, K. Weingarten, T. Ellermeyer, J. Lutz, M. Moeller, M. Huebner, J. Becker, C. Koos, W. Freude, and J. Leuthold, "26 Tbit s ${ }^{-1}$ line-rate super-channel transmission utilizing all-optical fast Fourier transform processing," Nature Photonics 5(6), pp. 364-371, 2011. 
[5] A. M. Weiner, "Femtosecond optical pulse shaping and processing," Progress in Quantum Electronics 19(3), pp. 161-237, 1995.

[6] Z. Jiang, D. S. Seo, D. E. Leaird, and A. M. Weiner, "Spectral line-by-line pulse shaping," Optics Letters 30(12), pp. 1557-1559, 2005.

[7] N. K. Fontaine, R. P. Scott, J. Cao, A. Karalar, W. Jiang, K. Okamoto, J. P. Heritage, B. H. Kolner, and S. J. B. Yoo, "32 phase32 amplitude optical arbitrary waveform generation," Optics Letters 32(7), pp. 865-867, 2007.

[8] A. E. Siegman, Lasers / Anthony E. Siegman, University Science Books, Mill Valley, Calif., 1986.

[9] Y. J. Kim, Y. Kim, B. J. Chun, S. Hyun, and S. W. Kim, "All-fiber-based optical frequency generation from an Er-doped fiber femtosecond laser," Optics Express 17(13), pp. 10939-10945, 2009.

[10] H. Y. Ryu, S. H. Lee, W. K. Lee, H. S. Moon, and H. S. Suh, "Absolute frequency measurement of an acetylene stabilized laser using a selected single mode from a femtosecond fiber laser comb," Optics Express 16(5), pp. 2867-2873, 2008.

[11] G. J. Schneider, J. A. Murakowski, C. A. Schuetz, S. Shi, and D. W. Prather, "Radiofrequency signalgeneration system with over seven octaves of continuous tuning," Nature Photonics 7(2), pp. 118-122, 2013.

[12] S. Fukushima, C. F. C. Silva, Y. Muramoto, and A. J. Seeds, "10 to $110 \mathrm{GHz}$ tunable opto-electronic frequency synthesis using optical frequency comb generator and uni-travelling-carrier photodiode," Electronics Letters 37(12), pp. 780-781, 2001.

[13] D. S. Wu, R. Slavik, G. Marra, and D. J. Richardson, "Robust optical injection locking to a $250 \mathrm{MHz}$ frequency comb without narrow-band optical pre-filtering," in 2011 Conference on Lasers and Electro-Optics (CLEO) Pacific Rim, pp. 273-275, 2011.

[14] S. Fukushima, C. F. C. Silva, Y. Muramoto, and A. J. Seeds, "Optoelectronic millimeter-wave synthesis using an optical frequency comb generator, optically injection locked lasers, and a unitraveling-carrier photodiode," Journal of Lightwave Technology 21(12), pp. 3043-3051, 2003.

[15] R. Lang, "Injection locking properties of a semiconductor-laser," IEEE Journal of Quantum Electronics 18(6), pp. 976-983, 1982.

[16] F. Mogensen, H. Olesen, and G. Jacobsen, "Locking conditions and stability properties for a semiconductorlaser with external light injection," IEEE Journal of Quantum Electronics 21(7), pp. 784-793, 1985.

[17] B. Kelly, R. Phelan, D. Jones, C. Herbert, J. O'Carroll, M. Rensing, J. Wendelboe, C. B. Watts, A. Kaszubowska-Anandarajah, P. Perry, C. Guignard, L. P. Barry, and J. O'Gorman, "Discrete mode laser diodes with very narrow linewidth emission," Electronics Letters 43(23), pp. 1282-1284, 2007. 